\title{
Untersuchungen zur Genetik des Diabetes mellitus. Eine geeignete Methode zur Durchführung von Alterskorrekturen.
}

\author{
J. KÖBBERLING \\ Institut für Humangenetik und Medizinischen Universitätsklinik, Göttingen
}

Eingegangen am 19. Mai 1969

\begin{abstract}
Investigations of the Genetics of Diabetes Mellitus. A suitable method for age correction.

Summary. A method for age correction, first described by Strömgren, and which can be applied to genetic investigations of diseases with varying age of onset, has been applied to an investigation of the geneties of diabetes mellitus. The main advantage compared with the often used method of "excess morbidity" is the fact that no investigation of control groups is necessary. It was found that the fre. quency of juvenile-onset diabetes among the siblings of juvenile-onset diabetics was about 25 times higher than that among the siblings of growth-onset diabetics. It must be concluded that there is genetic heterogeneity between juvenile and growth-onset diabetes. No significant difference could be found between the frequency of diabetes among siblings and that among the children of growthonset diabetics. This observation speaks in favour of preponderantly dominant inheritance of this type of diabetes.
\end{abstract}

Etude de la génétique du diabète sucré. Une méthode appropriée pour effectuer les corrections de l'âge

Résumé. L'auteur indique une méthode, déjà décrite par Strömgren, pour la correction de l'âge, qui peut être appliquée aux investigations génétiques de maladies qui apparaissent à des âges variés. L'avantage principal, par comparaison avec la méthode souvent employée de kexcess morbidity", est le fait qu'il n'est pas nécessaire d'avoir des groupes témoins. La méthode a été appliquée à l'étude de la génétique du diabète sucré. Il a été trouvé que la fréquence du diabète juvénile parmi les frères et soeurs de diabétiques juvéniles est environ 25 fois plus élevée que parmi les frères et soeurs de diabétiques âgés. On doit done conclure qu’il existe une hétérogénéité génétique entre le diabète juvénile et le diabète d'âge mûr. On n'a pas trouvé de différence significative entre la fréquence du diabète parmi les frères et soeurs ot los enfants des diabétiques d'âge mûr. Cette observation parle en faveur d'uno transmission essentiellement dominante de ce type do diabète.

Zusammenfassung. Es wird auf eine von Strömgren angegebene Methode zur Durchführung von Alterskorrekturen hingewiesen, die bei genetischen Untersuchungen von Krankheiten mit variierendem Erkrankungsalter Anwendung findet. Der wesentliche Vorteil gegenüber der häufig angewandten Methode der, ,excess morbidity" ${ }^{6} \mathrm{Be}$ rechnung liegt darin, daß keine Untersuchungen von Kontrollgruppen erforderlich sind. Bei Anwendung der Methode auf Untersuchungen zur Genetik des Diabetes mellitus fand sich, daß juveniler Diabetes unter den Geschwistern von juvenilen Diabetikern ca. 25 mal so häufig vorkommt wie unter den Geschwistern von Altersdiabetikern. Es besteht also eine genetische Heterogenität zwischen juvenilem Diabetes und Altersdiabetes. Zwischen der Diabeteshäufigkeit bei Geschwistern und Kindern von Altersdiabetikern besteht kein signifikanter Unterschied. Dieser Befund spricht für überwiegend dominante Vererbung des Altersdiabetes.

Key-words: Juvenile-onset diabetes, growth-onset diabetes, genetics, dominant inheritance, multifactorial inheritance, genetic heterogeneity, relatives of diabetics, frequency of diabetes, age correction.

\section{Einleitung}

Bei Untersuchungen zur Frage der Heterogenität des Diabetes mellitus wurde von verschiedenen Autoren $[1,9,20,21]$ eine von Penrose [17] angegebene Methode verwandt, bei der ohne Beruicksichtigung des Alters der Prozentsatz der erkrankten Geschwister (oder Eltern bzw. Kinder) ermittelt wird und mit einem entsprechenden Prozentsatz betroffener Geschwister aus einem ähnlich zusammengesetzten Vergleichskollektiv in Beziehung gesetzt wird. Das so gewonnene Ergebnis wird „K-value“ oder „excess morbidity“ genannt.

Die mit dieser Methode ermittelten Befunde zur Frage der Heterogenität zwischen juvenilem Diabetes und Altersdiabetes sind widerspruchsvoll. Keen $u$. Track [9] haben auf die große Unsicherheit der Methode und die möglichen Fehlerquellen hingewiesen. $\mathrm{Zu}$ den von diesen Autoren genannten Nachteilen der Methode seien nach folgende hinzugefügt:

1. Die Methode liefert nur dann exakte Ergebnisse, wenn Untersuchungs- und Kontrollgruppe sich in der altersmäBigen Zusammensetzung sehr ähnlich sind. Auch bezüglich aller anderen Faktoren, die die Morbidität beeinflussen könnten, muß die Zusammensetzung sehr ähnlich sein.

2. Der mittlere Fehler des berechneten Quotienten wird sehr groß, wenn die Kontrollgruppe, bei der in der Regel eine geringe Morbiditätsrate zu erwarten ist, zu klein ist.

3. Da die Berücksichtigung bereits verstorbener Geschwister Schwierigkeiten bereitet, geht oft ein Teil der Informationen verloren.

Es soll deshalb eine andere Methode zur Alterskor. rektur erläutert werden, die erstmals von Strömgren [25] bei Untersuchungen zur Genetik der Schizophrenie angegeben wurde. Die technische Durchführung der Berechnung wurde leicht abgewandelt. Der Vorteil der Strömgren-Methode liegt in einer größeren Genavigkeit und Anschaulichkeit der Ergebnisse und der Möglichkeit, ohne eine Kontrollgruppe auszukommen. Dafür muß ein etwas größerer Rechenaufwand in Kauf genommen werden. Als Beispiel soll ebenfalls die Frage 
der genetischen Heterogenität zwischen juvenilem Diabetes und Altersdiabetes sowie die Frage einer unterschiedlichen Belastung von Geschwistern und Kindern von Altersdiabetikern geprüft werden.

\section{Probanden und Methode}

583 zufällig ausgewählte Diabetiker aus der Diabetes-Ambulanz der Medizinischen Universitätsklinik Göttingen wurden nach dem Vorkommen von Diabetes unter ihren Eltern, Geschwistern und Kindern befragt. Ohne Berücksichtigung des klinischen Typs wurden alle Diabetiker mit einem Beginn der Erkrankung bis zum 25. Lebensjahr als juvenile Diabetiker bezeichnet (35 Probanden), alle später erkrankten als Altersdiabetiker (548 Probanden). Die Grenze von 25 Jahren ergibt sich aus den Vorschlägen der WHO zur Einteilung der Diabetes-Typen [26]. Obgleich das Er. krankungsalter der betroffenen Angehörigen häufig nicht genau zu ermitteln war, konnte die Einteilung in vor und nach dem 25. Lebensjahr Erkrankte auch bei ihnen mit ausreichender Sicherheit vorgenommen werden. Die Zusammensetzung des Ausgangskollektivs nach dem Alter bei der Diagnosestellung, zusammengefaßt in 5 Jahresgruppen, zeigt die Abb. 1.

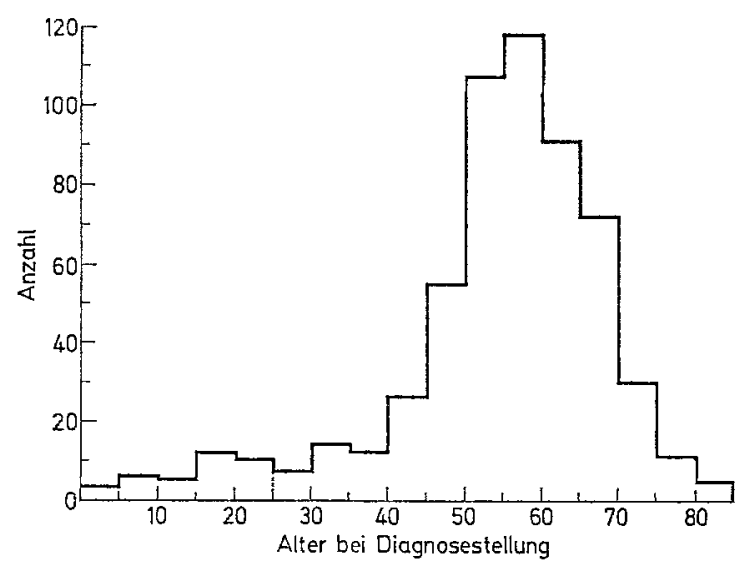

Abb. 1. Verteilung der Ausgangsfälle nach dem Alter bei Diagnosestellung, zusammengefaßt in 5-Jahres-Gruppen

Die hier verwandte Methode zur Alterskorrektur soll am Beispiel des Altersdiabetes erläutert werden (Tabelle 1). Die Ausgangsfälle werden nach dem Erkrankungsalter (Spalte 1) in 5-Jahresgruppen zusammengefaßt (Spalte 2). Die Zahl der in jedem Altersabschnitt Erkrankten wird durch die Gesamtzahl der Personen des entsprechenden Alters in der Allgemeinbevölkerung (Spalte 3) geteilt. (Das Jahr 1961 ergibt sich aus dem durchschnittlichen Erkrankungszeitpunkt der Probanden). Die so gewonnenen Quotienten (Spalte 4) werden addiert und jeder einzelne wird in Prozent der Gesamtsumme angegeben (Spalte 5). So ergeben sich für jede Altersklasse die Werte der relativen Erkrankungswahrscheinlichkeit. Diese werden schlieBlich fortlaufend aufsummiert (Spalte 6). Die so ermittelten Endwerte geben an, wieviel Prozent aller Personen, die bis zum Ende der Gefährdungsperiode erkrankt sein werden, bei einem bestimmten Alter bereits erkrankt sind.

Tabelle 1. Berechnung der relativen Erkrankungswahrscheinlichkeit für Aliersdiabetes in Abhängigkeit vom Alter

\begin{tabular}{lrlrrr}
\hline 1 & 2 & 3 & \multicolumn{1}{l}{4} & \multicolumn{1}{c}{5} & \multicolumn{1}{c}{6} \\
\hline $26-30$ & 7 & 3.598 & 1.95 & 1.0 & 1.0 \\
$31-35$ & 14 & 3.701 & 3.78 & 1.8 & 2.8 \\
$36-40$ & 12 & 3.721 & 3.22 & 1.6 & 4.4 \\
$41-45$ & 27 & 2.442 & 11.06 & 5.4 & 9.8 \\
$46-50$ & 55 & 3.818 & 14.41 & 7.1 & 16.9 \\
$51-55$ & 107 & 3.880 & 27.58 & 13.6 & 30.5 \\
$56-60$ & 118 & 3.533 & 33.40 & 16.4 & 46.9 \\
$61-65$ & 91 & 2.792 & 32.59 & 16.0 & 62.9 \\
$66-70$ & 72 & 2.090 & 34.45 & 17.0 & 79.9 \\
$71-75$ & 29 & 1.540 & 18.83 & 9.3 & 89.2 \\
$76-80$ & 11 & 0.958 & 11.48 & 5.6 & 94.8 \\
$81-85$ & 5 & 0.473 & 10.57 & 5.2 & 100.0 \\
über 85 & 0 & 0.155 & 0.00 & 0.0 & 100.0 \\
& & Summe: & 203.32 & & \\
\hline
\end{tabular}

Spalte 1: Lebensalter in 5-Jahresgruppen

Spalte 2: Anzahl der in den. Altersstufen neu entdeckten Diabetiker

Spalte 3: Anzahl der Personen dieser Altersstufe in der Allgemeinbevölkerung in 1000000 (Stat. Jahrb. d. BRD, 1961)

Spalte 4: Quotient der Einzelwerte aus Sp. 2 und 3

Spalte 5: Prozentsatz der Einzelwerte aus Sp. 4 von der Summe aus Sp. 4

Spalte 6: Fortlaufende Aufsummierung der Werte aus Sp. 5

Bei der Auszählung der Geschwister wird jede Person mit dem Zahlenwert eingesetzt, der sich aus dieser Berechnung ergibt. Wenn auch die Geschwister in 5Jahresgruppen zusammengefaßt werden, dann muß aus jeweils 2 Werten der Mittelwert gebildet werden, da die Werte der Spalte 6 der Tabelle 1 sich auf das Ende einer 5-Jahresperiode beziehen. Für verstorbene Geschwister wird der Wert des erreichten Lebensalters eingesetzt. Das so gewonnene Ergebnis besagt nichts über die Zahl der aktuell erkrankten Geschwister sondern darüber, wie viele Geschwister an Diabetes erkranken würden, wenn alle eine bestimmte Gefähr. dungszeit voll durchlaufen würden.

\section{Ergebnisse}

Die Werte der relativen Erkrankungswahrscheinlichkeit wurden nach der oben angegebenen Methode getrennt für juvenilen Diabetes und Altersdiabetes berechnet (Abb. 2). Die Punkte der Kurve des Altersdiabetes (ausgezogene Linie) ergeben sich aus der Tabelle 1. Für juvenilen Diabetes wurde eine gleichartige Berechnung durchgeführt. Hier lautet der Wert vom 25. Lebensjahr ab definitionsgemäß 1 (gestrichelte Kurve).

Für die 35 juvenilen Diabetiker errechnet sich unter Zugrundelegung der gestrichelten Kurve der Abb. 2 eine „reduzierte" Gesamtzahl von 64.0 Geschwistern. 
Davon hatten 7 vor Vollendung des 25. Lebensjahres einen Diabetes bekommen. Die Geschwister von juvenilen Diabetikern haben also eine Chance von $10.9 \pm$ $3.9 \%$ bis zum 25. Lebensjahr an Diabetes zu erkranken. Für die 548 Altersdiabetiker errechnet sich nach der

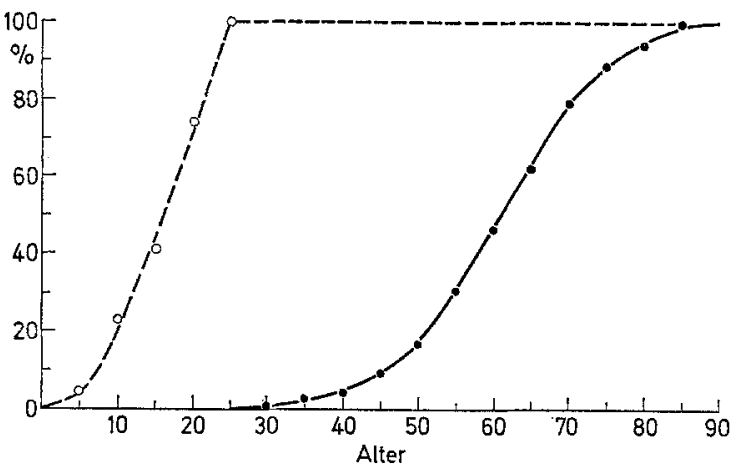

Abb. 2. Kurven der relativen Erkrankungswahrscheinlichkeit der einzelnen Altersstufen in Prozent der Gesamtwahrscheinlichkeit.

Gestrichelte Kurve = juveniler Diabetes, durchgezogene Kurve $=$ Altersdiabetes

gestrichelten Kurve eine ,,reduzierte“ Gesamtzahl von 2004.5 Geschwistern, von denen 8, das sind $0.4 \pm 0.1 \%$, einen juvenilen Diabetes hatten. Juveniler Diabetes ist also unter den Geschwistern der juvenilen Diabetiker ca. 25mal so häufig wie unter den Geschwistern der Altersdiabetiker. Bemerkenswert ist, daß von den 8 hier als Altersdiabetiker eingestuften Probanden mit einem juvenildiabetischen Geschwister 4 vor dem 35 . Lebensjahr erkrankt waren und insulinbedürftig sind, also eigentlich dem Typ des ,juvenile onset diabetes" zuzurechnen sind.

Unter Zugrundelegung der rechten Kurve der Abb. 2 ergibt sich für die Altersdiabetiker eine reduzierte Gesamtzahl von 833.3 Geschwistern, von denen 215 einen Altersdiabetes hatten. Daraus errechnet sich, daß durchschnittlich $25.8 \pm 1.5 \%$ der Geschwister von Altersdiabetikern zwischen dem 26. und 85. Lebensjahr an Diabetes erkranken werden. Leider ist die Zahl der älteren Geschwister der juvenilen Diabetiker so gering, daß sich keine Aussage über die Häufigkeit des Altersdiabetes unter ihnen machen läßt.

Nur zwei Elternteile der juvenilen Diabetiker waren an Altersdiabetes erkrankt. Da die reduzierte Gesamtzahl 23.5 beträgt, sind $8.5 \pm 5.6 \%$ der Eltern jureniler Diabetiker Altersdiabetiker. Nur ein Elternteil von 64 war an juvenilem Diabetes erkrankt $=$ $1.6 \pm 1.3 \%$.

Eine entsprechende Berechnung wurde für die Kinder der Altersdiabetiker durchgeführt. Dabei ergab sich, daß 3 von $1111.9=0.3 \pm 0.2 \%$ der Kinder bis zum 25. Lebensjahr an Diabetes erkranken und 18 von $54.0=33.4 \pm 6.4 \%$ zwischen dem 26 . und 85. Lebensjahr. Der Unterschied zu den Belastungsziffern für Geschwister ist nicht signifikant. Die Ergebnisse sind in Tabelle 2 noch einmal zusammengestellt.

\section{Diskussion}

Bei genetischen Untersuchungen von Merkmalen mit variierendem Erkrankungsalter sind Alterskorrekturen erforderlich. Der besondere Vorteil der hier geschilderten Strömgren-Methode liegt darin, daß zur Berechnung der Belastungsziffern keine Untersuchungen von Kontrollgruppen notwendig sind. Es wird dabei davon ausgegangen, daß die Altersverteilung der Neuerkrankungen bei den zu untersuchenden Verwandten derjenigen der Probanden entspricht. Die Methode läßt sich also nicht anwenden, wenn das Ausgangskollektiv nicht auslesefrei ausgewählt wurde, sondern nach irgendwelchen Eigenschaften, die die Alterszusammensetzung verändern. Man könnte jedoch in einem solchen Fall auf eine aus einem anderen repräsentativen Kollektiv errechnete Kurve der relativen Erkrankungswahrscheinlichkeit zurückgreifen. Eskann natürlich auch nicht als sicher angenommen werden, daß die Diabetiker, die sich zu ihrer Betreuung in die Diabetes-Ambulanz begeben haben, bezüglich des Erkrankungsalters repräsentativ sind. Der Fehler, der sich aus einer nicht ganz korrekten Alterskurve ergeben würde, ist für die Aussagen der vorliegenden Arbeit allerdings unerheblich, da jeweils verschiedene Kollektive miteinander verglichen werden, deren Geschwisterbelastung nach der gleichen Kurve berechnet wurde.

Die Berechnungen mit der geschilderten Methode geben keine Auskunft über die Anzahl der zum Zeitpunkt der Untersuchung erkrankten Geschwister (oder Eltern bzw. Kinder), sondern über die Anzahl derer, die vermutlich erkranken würden, wenn alle ein bestimmtes Alter erreichen würden. Dadurch ist das Ergebnis unabhängig von der augenblicklichen Alterszusammensetzung des Ausgangskollektivs und der Verwandten.

Die Unterteilung in juvenile Diabetiker und Altersdiabetiker ausschließlich nach dem Erkrankungsalter wird der klinischen Typeneinteilung in den insulinbedürftigen zu Ketose neigenden, ,juvenile onset type und den häufig mit Diät oder oralen Antidiabetika einstellbaren nicht zur Ketose neigenden ,growth onset type " nicht gerecht. Die hier angewandte Methode setzt aber eine fixierte Altersgrenze voraus, solange nicht auch bei den betroffenen Familienangehörigen in jedem Einzelfall eine Zuordnung zu einem dieser Typen möglich ist. Bei einer neuen Untersuchungsserie streben wir eine solche, den klinischen Gesichtspunkten gerecht werdende Unterteilung, an.

Die obengenannten Untersuchungen zur genetischen Heterogenität des Diabetes [1, 9, 20, 21] gehen von der Frage aus, ob juveniler Diabetes ,,stärker erblich" ist als Altersdiabetes. Die Frage der vorliegenden Arbeit lautet dagegen, ob juveniler Diabetes unter den Geschwistern der juvenilen Diabetiker häufiger ist als unter den Geschwistern der Altersdiabetiker. Der Unterschied von $10.9 \pm 3.9$ zu $0.4 \pm 0.1$ ist hoch signifikant. Das Ergebnis ließe den Schluß zu, daß es sich bei juvenilem Diabetes und Altersdiabetes um zwei gene- 
tisch verschiedene Krankheiten handelt, wobei sich allerdings die Erkrankungsalter beider Typen in einem gewissen Bereich überschneiden. Für diese Annahme spricht, daß juveniler Diabetes unter den Geschwistern und Kindern der Altersdiabetiker kaum häufiger als in der Allgemeinbevölkerung ist.

Andere Autoren [2, 7] meinten, daß ein einziges Gen in homozygoten Zustand zu juvenilem Diabetes führen könnte, in heterozygotem Zustand jedoch zu Altersdiabetes. Dann müßte unter den Geschwistern der juvenilen Diabetiker Altersdiabetes sehr häufig sein. Zur Beantwortung dieser Frage reicht das vorliegende Material nicht aus. Es müßten außerdem sämt. ermitteln [10]. Hierin ist eine weitere Stütze für die Annahme dominanter Vererbung zu sehen. Zusammenfassend läßt sich sagen, daß die Zahlen der Tabelle 2 folgende Aussagen erlauben:

1. Bezüglich der Geschwisterbelastung mit juvenilem Diabetes besteht ein signifikanter Unterschied zwischen juvenilen Diabetikern und Altersdiabetikern.

2. Die Diabetesbelastung ist bei Geschwistern und Kindern von Altersdiabetikern nicht signifikant verschieden.

3. Die Belastung mit Altersdiabetes ist bei den Eltern der juvenilen Diabetiker signifikant niedriger als bei Geschwistern und Kindern von Altersdiabetikern.

Tabelle 2. Belastung mit juvenilem Diabetes und Altersdiabetes nach Alterskorrekturen für Eltern, Geschwister und Kinder von juvenilen Diabetikern und Altersdiabetikern

\begin{tabular}{l|l|l|l|l}
\hline Ausgangsfälle & Belastung mit & Eltern & Geschwister & Kinder \\
\hline \multirow{2}{*}{ juvenile Diabetiker } & juvenilem Diabetes & $\mathbf{1 . 6 \pm 1 . 3 \%}$ & $\mathbf{1 0 . 9 \pm 3 . 9 \%}$ & - \\
\cline { 2 - 6 } & Altersdiabetes & $\mathbf{8 . 5 \pm 5 . 6 \%}$ & - & - \\
\hline \multirow{2}{*}{ Altersdiabetiker } & juvenilem Diabetes & - & $0.4 \pm 0.1 \%$ & $0.3 \pm 0.2 \%$ \\
\hline
\end{tabular}

liche Eltern der juvenilen Diabetiker heterozygote Genträger, also potentielle Altersdiabetiker sein. Es fanden sich unter ihnen jedoch auch bei Berücksichtigung von Alterskorrekturen nur 8.5\% Altersdiabetiker.

Schließlich wäre zu diskutieren, ob es sich nur um ein Diabetesgen handeln könnte, bei dem aber Nebengene in bestimmten Familien zu einer Ausprägung der Krankheit in früherem Lebensalter führen. Diese Deutung kommt der Annahme multifaktorieller Vererbung $[8,12,19,20,21]$ sehr nahe. Der erhebliche Unterschied in der Geschwisterbelastung von 1:25 wäre dabei allerdings schwer vorstellbar. Auch wäre in diesem Fall zu erwarten, daß von den Eltern der juvenilen Diabetiker ein höherer Prozentsatz an Diabetes erkrankt. Mit der These eines einzigen recessiven Gens für alle klinisch unterschiedlichen Diabetestypen $[6,13,16$, 22, 23, 24] sind die vorliegenden Befunde nicht vereinbar.

Der Vergleich der Diabetesbelastung unter den Geschwistern und den Kindern der Altersdiabetiker erlaubt Rückschlüsse auf den Erbgang dieses DiabetesTyps. Bei recessiver Vererbung wäre z.B. bei einer Genhäufigkeit von 5\% zu erwarten, daß 22\% der Kinder und 37\% der Geschwister von Diabetikern homozygot betroffen sind [3]. Die Beobachtung einer etwa gleich starken Belastung der Gesohwister und der Kinder von Altersdiabetikern spricht für' eine unregelmäBig dominante Vererbung, wie sie auch von anderen Autoren entweder für alle Diabetes-Typen $[4,5,11$, $14,15,18]$ oder nur für den Altersdiabetes [2, 7] angenommen wird. Unter Einbeziehung der bei Durchführung eines oralen Glucosetoleranztests entdeckten latenten oder subklinischen Diabetiker konnten wir für die Geschwister eine Belastungsziffer von ca. 50\%
Unsere bisher geringen Kenntnisse über die Genetik des Diabetes mellitus lassen sich wie folgt zusammenfassen: Der Diabetes ist genetisch heterogen. Am Altersdiabetes scheint ein vorwiegend dominanter Geneffekt beteiligt zu sein, beim juvenilen Diabetes spielt möglicherweise Recessivität eine Rolle.

\section{Literatur}

1. General Practitioners, Report of a Working Party: The family history of diabetics. Brit. med. J. 1965 I, $960-962$.

2. Grunnet, J.: Heredity in diabetes mellitus. A proband study. Opera ex Domo Biologiae Hereditariae Humanae Universitatis Hafniensis, Vol. 39, 1-128. Copenhagen: Munksgaard, 1957.

3. Grüttner, R., Lenz, W.: Genetisch bedingte Störungen des Kohlenhydratstoffwechsels. Z. menschl. Vererb.- u. Konstit.-Lehre 36, 265-287 (1962).

4. Günther, O.: Zur Genetik von Diabetes mellitus und renalem Diabetes. Med. Kl. 56, 835-837 (1961).

5. - Probleme der Genetik des Diabetes mellitus. Internist 4, 374-384 (1963).

6. Hanhart, E.: Nachweis der ganz vorwiegend einfachrezessiven Vererbung des Diabetes mellitus. Erbarzt 6, $5-15(1939)$.

7. Harris, H. : The familial distribition of diabetes mellitus: a study of the relatives of 1241 diabetic propositi. Ann. Eugen. 15, 95-119 (1950).

8. Jörgensen, G.: Zur Genetik des idiopathischen Diabetes mellitus. Deutsch. med. J. 17, 609-616 (1966).

9. Keen, H., Track, N. S.: Age of onset and inheritance of diabetes. The importance of examining relatives. Diabetologia 4, 317-321 (1968).

10. Köbberling, J., Appels, A., Köbberling, G. Creutzfeldt, W.: Glucosebelastungstests bei 727 Verwandten ersten Grades von Altersdiabetikern. Dtsch. med. Wschr. 94, 416-421 (1969).

11. v. Kries, I.: Beitrag zur Genetik des Diabetes mellitus. Z. menschl. Vererb.-u. Konstit.-Lehre 31, 406-430 (1953). 
12. Neel, J.V., Fajans, S.S., Conn, J.W., Davidson, R.T.: Diabetes mellitus. In: Genetics and the epidemiology of chronic diseases. Edited by J.V. Neel, E.W. Shaw, W.J. Schull. Public Health Service Publication No. 1163, S. $105(1965)$.

13. Nilsson, S.E.: On the heredity of diabetes mellitus and its interrelationship with some other diseases. Acta genet. et statist. med. (Basel) 14, 97-124 (1964).

14. Pavel, I., Pieptea, R.: On the mode of transmission of hereditary diabetes. Diabetologia 4, 358-364 (1968).

15. - - Etude sur le diabète héréditaire au cours de 3 et 4 générations successive. Diabetologia 2, $281-285$ (1966).

16. Penrose, L.S.: The genetical background of common diseases. Acta genet. (Basel) 4, 257-265 (1953).

17. Post, R.H.: An approach to the question, does all diabetes depend upon a single genetic locus? Diabetes $\mathbf{1 1}$ $56-65$ (1962).

18. Schade, H.: Bemerkungen über , unregelmäßige Dominanz" am Beispiel des Diabetes mellitus. Medizinische, $595-598$ (1958).

19. Simpson, N.E.: The geneties of diabetes: A study of 233 families of juvenile diabetics. Ann. hum. Genet, (London) 26, 1-21 (1962).
20. - Multifactorial inheritance. A possible hypothesis for diabetes. Diabetes 13, 462-471 (1964).

21. - Diabetes in the families of diabetics. Vortrag VI. Congr. Intern. Diabetes Fed. Stockholm 1967.

22. Steinberg, A.G.: Heredity and diabetes. Eugen. Quart. 2, 26 (1955).

23. - Genetics and diabetes. In: On the nature and treatment of diabetes. Amsterdam: Excerpta medica foundation, 1965 .

24. - Wilder, R.M.: A study of the genetics of diabetes mellitus. Amer. J. Human. Genet. 4, 113-135 (1952).

25. Strömgren, E.: Zum Ersatz des Weinberg'schen ,abgekürzten Verfahrens". Zugleich ein Beitrag zur Frage von der Erblichkeit des Erkrankungsalters bei der Schizophrenie. Zbl, ges. Neurol. Psychiat. 153, $784-797$ (1935).

26. WHO-Expert-Committee: Diabetes mellitus. No. 310. Genf: Wld. Flth. Org. techn. Rep. Ser. 1965.

Dr. J. Köbberling

Med. Univ.-Klinik

34 Göttingen

Humboldtallee 1 